\title{
Early calcium dysregulation in Alzheimer's disease: setting the stage for synaptic dysfunction
}

\author{
Shreaya CHAKROBORTY \& Grace E. STUTZMANN* \\ Department of Neuroscience, Rosalind Franklin University/the Chicago Medical School, North Chicago IL 60064, USA
}

Received May 15, 2011; accepted May 30, 2011

\begin{abstract}
Alzheimer's disease (AD) is an irreversible and progressive neurodegenerative disorder with no known cure or clear understanding of the mechanisms involved in the disease process. Amyloid plaques, neurofibrillary tangles and neuronal loss, though characteristic of $\mathrm{AD}$, are late stage markers whose impact on the most devastating aspect of $\mathrm{AD}$, namely memory loss and cognitive deficits, are still unclear. Recent studies demonstrate that structural and functional breakdown of synapses may be the underlying factor in AD-linked cognitive decline. One common element that presents with several features of AD is disrupted neuronal calcium signaling. Increased intracellular calcium levels are functionally linked to presenilin mutations, ApoE4 expression, amyloid plaques, tau tangles and synaptic dysfunction. In this review, we discuss the role of AD-linked calcium signaling alterations in neurons and how this may be linked to synaptic dysfunctions at both early and late stages of the disease.
\end{abstract}

calcium, Alzheimer's, neuron, synaptic dysfunction, plasticity, ER, ryanodine, CICR

Citation: Chakroborty S, Stutzmann G E. Early calcium dysregulation in Alzheimer's disease: setting the stage for synaptic dysfunction. Sci China Life Sci, 2011, 54: 752-762, doi: 10.1007/s11427-011-4205-7

\section{Alzheimer's disease and the relevance of synaptic dysfunction}

Alzheimer's disease (AD) is a devastating neurodegenerative disorder that gradually and insidiously destroys neurons, leading to cognitive, memory and behavior impairments. $\mathrm{AD}$ is definitively diagnosed through patient history of cognitive decline in conjunction with postmortem findings of amyloid beta $(\mathrm{A} \beta)$ plaques, neurofibrillary tau tangles and extensive neuronal loss in the hippocampus and neocortex. The most common form, termed sporadic $\mathrm{AD}$, has a relatively late age of onset ( $>65$ years of age), though its etiology remains a mystery. The inherited or familial form (FAD) exhibits a similar pattern of cognitive decline and histopathology, though the progression of symptoms is far more

*Corresponding author (email: grace.stutzmann@ rosalindfranklin.edu) aggressive and the age of onset is markedly younger (30-40 years). FAD is linked to mutations in the amyloid precursor protein (APP) and the presenilin (PS) 1 and 2 genes. While the mechanistic link between the development of $\mathrm{AD}$ and these gene mutations is unclear, their expression will invariably lead to $\mathrm{AD}[1,2]$.

The amyloid beta peptide, the primary component of plaques, is derived from altered processing of the amyloid precursor protein (APP). Sequential cleavage of APP by $\beta$ and $\gamma$-secretases results in production of $A \beta$ peptides-the more common $A \beta_{40}$ and the pathogenic $A \beta_{42}$. Presenilin forms the catalytic core of the $\gamma$-secretase enzyme complex and regulates the intramembranous cleavage of APP to $A \beta$. The majority of presenilin gene mutations studied in model systems have been shown to alter $\gamma$-secretase cleavage of APP, resulting in increased production of the toxic $\mathrm{A} \beta_{42}$ [2-4]. These same presenilin mutations have also been 
linked to altered intracellular calcium signaling in a variety of model and cell systems, and will be the subject of further discussion later in this review.

Despite being an obligatory diagnostic feature of $\mathrm{AD}, \mathrm{A} \beta$ and tau deposits are poorly correlated with the most devastating aspect of $\mathrm{AD}$, namely the progressive memory loss and cognitive decline. In fact, both $\mathrm{A} \beta$ plaques and tau tangles are present in the hippocampus and neocortex of individuals without cognitive deficits or dementia, at levels similar to those found in individuals with dementia [5-8]. More recent and convincing evidence implicates the breakdown of synaptic integrity and function as a better correlate, and likely causative agent, of cognitive decline in AD [9-14]. These studies demonstrate not only neuronal loss but a reduction in dendritic arborization and dendritic spine density of pyramidal neurons in AD patients and animal models. Indeed, AD has increasingly been referred to as a "synaptic disease" because of the central role synaptic breakdown has in the devastating cognitive deficits of the disease [10,15-17]. Although this review will focus on the proximal role calcium dysregulation has in promoting synaptic dysfunction in $\mathrm{AD}$, it also is worth noting that other hallmark, but not exclusive, features of AD such as $A \beta$ and hyperphosphorylated tau, also introduce synaptic pathology (as well as calcium dysregulation) [18-21]. It is likely that the combined, feed-forward pathogenic cascades among these features will accelerate the demise of synapses in AD, though independently each can result in dysfunction.

There are several reasons why synaptic impairment is of principal significance to cognitive loss in $\mathrm{AD}$. One of the observations in the brains of $\mathrm{AD}$ patients is a reduction in the number of dendritic spines and their connections with presynaptic terminals to anchor a functional synapse [11-14]. Structurally, the dendrites of pyramidal neurons are covered with numerous spines, or protrusions, that serve as the postsynaptic sites for most excitatory synapses. Within these small compartments $\left(\sim 0.01\right.$ to $\left.0.8 \mu^{3}\right)$, an extensive array of cellular machinery is condensed which supports a unique signaling microcosm of receptors, scaffolding proteins, second messengers, kinases/phosphatases, gene transcription factors and immediate early genes, and, among other signaling factors, calcium [22,23]. Here, calcium serves many functions, but the common denominator is its role in transducing and modifying neurochemical signals released from presynaptic terminals into electrical gradients (such as through voltage-gated calcium channels, and calcium-permeable postsynaptic receptors), and promoting downstream signaling cascades by activating calciumsensitive kinases, phosphatases, and the like [24,25]. Because of the unique geometry of the spine head and neck, which confers a high resistance barrier to the adjacent dendritic process, the spine head signaling domain is protected to maintain proper synaptic transmission and plasticity properties, which in turn can support learning and memory functions within key brain regions [22,23]. Therefore, lo- calized calcium signals in dendritic spines and processes are central to proper neuronal activity and synaptic functioning, and sustained calcium dyshomeostasis can alter both the structural and functional aspects of the spine apparatus [26,27]. This can occur in either the absence of amyloid or tau histopathology, or, become greatly accelerated in the presence of misfolded protein aggregates.

\section{AD pathology, risk factors, and mechanisms of early calcium dyshomeostasis}

Maintenance of intracellular calcium homeostasis is essential for the functioning and survival of neurons and is a fundamental component of synaptic transmission for both pre- and postsynaptic mechanisms [28,29]. Given the crucial and widespread roles calcium signaling has in excitable cells, it is not surprising that alterations in calcium homeostasis are linked to several neurodegenerative conditions [30-33]. Calcium concentrations in the cytosol are typically maintained at nanomolar levels but can locally be increased to micromolar levels within distinct microdomains such as outside channel pore regions and within enclosed domains such as spine heads $[22,34]$. Cytosolic calcium entry is predominantly mediated by ligand-gated channels (such as NMDA receptors) or voltage-gated calcium channels (VGCC) located in the plasma membrane. Release of calcium from intracellular ER sources occurs via ER membrane channels-inositol trisphosphate receptor $\left(\mathrm{IP}_{3} \mathrm{R}\right)$ and ryanodine receptor $(\mathrm{RyR})$. The $\mathrm{IP}_{3} \mathrm{Rs}$ are activated by the second messenger $\mathrm{IP}_{3}$ while the RyRs are activated by cytosolic calcium. The activation of both of these channels is augmented by the phenomenon of calcium-induced calcium release (CICR) a regenerative process in which calcium enhances its own release from $\mathrm{IP}_{3} \mathrm{R}$ and RyR. This self-limiting dynamic enables the recruitment of ER calcium through a variety of calcium signaling pathways in neuronal physiology, serving in part as a coincidence detector across calcium sources. For instance, RyR-mediated CICR can be augmented by calcium entering through plasma membrane calcium channels as well as calcium released through the $\mathrm{IP}_{3} \mathrm{R}$ [35-37]. Mitochondrial calcium dynamics make a relatively smaller contribution, but are still an important component in regulating calcium homeostasis by quickly taking up excess calcium released from ER stores and slowly releasing it in the cytosol [38].

Aberrant neuronal calcium signaling is increasingly implicated as a pathogenic factor in $\mathrm{AD}$ and is also observed with sporadic AD risk factors such as ApoE 4 expression and CALHM1 polymorphisms (Figure 1). The calcium dyshomeostasis resulting from AD-linked PS mutations, as well as presumed ApoE4 expression and CALHM polymorphisms, is present throughout the organism's lifetime $[32,39,40]$. This is in contrast to the emergence of amyloid plaques and tau tangles relatively late in one's lifetime-in 
both neurodegenerative disease states and as a part of normal aging. In familial and sporadic AD associated with genetic risk factors, the long-term exposure to genetic aberrations suggests that either cumulative lifelong challenges, or predisposing vulnerabilities to age-dependent or epigenetic insults, may contribute to the pathology resulting in $\mathrm{AD}$. However, identifying the actual early pathogenic mechanisms has thus far been elusive save for increased ER calcium linked to PS mutations.

The effects of PS mutations on calcium dysregulation have been demonstrated in model cell systems, namely transgenic mice and human cells [41-46]. Several mechanisms have been proposed for the effects of mutant PS on ER calcium signaling. One hypothesis suggests that PS, in its wild-type state, may function as an ER leak channel that maintains ER calcium homeostasis by releasing calcium and balancing SERCA (Sarco/Endoplasmic Reticulum Calcium ATPase) pump activity. Mutant PS may be impaired as a leak channel, and as a result, ER calcium stores become overfilled which can introduce homeostatic changes in RyR expression, protein misfolding, and other downstream consequences $[47,48]$. Direct alterations of $\mathrm{IP}_{3} \mathrm{R}$ function have also been documented. Mutant PS1 has been shown to increase the open probability of the $\mathrm{IP}_{3} \mathrm{R}$ and to shift modal gating properties to bursts of repetitive openings at what would normally be sub-threshold $\mathrm{IP}_{3}$ concentrations, resulting in net increased ER calcium release through these channels $[49,50]$. Consistent with this, within the soma of mutant PS1-expressing neurons, baseline circulating levels of cytosolic $\mathrm{IP}_{3}$ are sufficient to generate a modest $\mathrm{IP}_{3}$-evoked calcium response in the presence of elevated calcium (a necessary co-agonist at the $\mathrm{IP}_{3} \mathrm{R}$ ), whereas in control neurons, this low-level $\left[\mathrm{IP}_{3}\right]$ is insufficient to activate the $\mathrm{IP}_{3} \mathrm{R}[16]$.

There are also several lines of evidence demonstrating effects of mutant PS on RyR-mediated calcium release and CICR threshold. In mutant PS-expressing animal models, increased RyR-mediated calcium release is observed before the histopathological and cognitive symptoms emerge and persists throughout the organism's lifetime [46,51]. Additionally, conditions which result in increased ER calcium levels can further enhance RyR responsivity to cytosolic calcium [52,53], thereby augmenting CICR and establishing a long-term feed-forward mechanism of calcium cycling. Direct modulation of RyR by PS may be one concrete mechanism by which ER calcium dyshomeostasis results. The PS1 N-terminal domain is thought to bind to and potentiate RyR calcium flux, and under normal conditions, the degree of calcium release is kept within physiological limits by a calcium-dependent negative feedback mechanism by which the RyR detects elevated calcium and subsequently suppresses further calcium release [54]. Mutant PS1 may impair this feedback function whereby RyR activity persists even at high calcium concentration. There is also evidence that PS1 mutations increase ryanodine receptor (RyR) ex- pression levels in neurons $[46,51,55]$, with distinct isoform specific patterns. At early or presymptomatic stages, the RyR2 isoform is selectively upregulated and may underlie the exaggerated RyR-evoked calcium responses and sensitized CICR, while at later stages, coincident with $\mathrm{A} \beta_{42}$ deposition, RyR3 levels upregulate in what is possibly a neuroprotective response [16,56-58]. The RyR2 is densely expressed in several brain regions vulnerable in $\mathrm{AD}$, especially the hippocampus, while the RyR3 expression patterns are typically more diffuse and relatively sparse $[59,60]$. Notably, increased RyR2 expression is also observed in individuals with mild cognitive impairment (MCI), which is thought to be an early stage of dementia that ultimately will convert to AD [61].

Resulting from this preexisting calcium dysregulation, a subsequent pathogenic cascade may develop between increased calcium levels and $\mathrm{A} \beta$ deposition in $\mathrm{AD}$. $\mathrm{A} \beta$ oligomers may increase calcium influx by forming calcium pores in the plasma membrane and by regulating existing plasma membrane calcium channels [62-64]. Increased intracellular calcium, and more specifically, RyR-mediated calcium release, can in turn facilitate the formation of pathogenic A $\beta$ peptides and plaque aggregation [65-67], and equally increasing $A \beta$ load can increase RyR3 expression [57]. An analogous facilitatory pathogenic relationship may exist between calcium signaling and tau pathology. Increased intracellular calcium potentiates neurofibrillary tangle formation by upregulating the activity of calcium-dependent tau kinases such as glycogen synthase kinase $3 \beta$, protein kinase $\mathrm{C}, \mathrm{cdk} 5$ and others [68]. Conversely, hyperphosphorylated tau can further raise intracellular calcium concentrations by compromising the integrity of neuronal processes, altering signaling cascades, and upregulating cholinergic receptor activation coupled to calcium release [32].

The known sporadic AD risk factors such as CALHM1 and ApoE4 allele expression also alter intracellular calcium levels. The CALHMI gene encodes a transmembrane calcium permeable channel which is inserted in ER and plasma membranes. Recently, a polymorphism of CALHM1 was found that is associated with increased AD risk, increasing $\mathrm{A} \beta$ deposition by altering calcium permeability [40]. Apolipoprotein E (ApoE) is a lipid-associated protein involved in transport of lipids. There are three ApoE alleles, ApoE2, ApoE3 and ApoE4, each conferring distinct vulnerability to AD. Expressing ApoE2 is considered neuroprotective, while $\mathrm{E} 4$ expression is linked to increased risk of AD [69,70]. ApoE4 increases intracellular calcium levels via a cell-surface LDL receptor-mediated process that recruits plasma membrane calcium channels as well as RyR-mediated ER stores [32]. Further, ApoE4 is found in close proximity to amyloid plaque, suggesting that it may play a role in $A \beta$ formation and in a larger pathogenic process involving lipid metabolism, calcium, and protein folding [71]. 


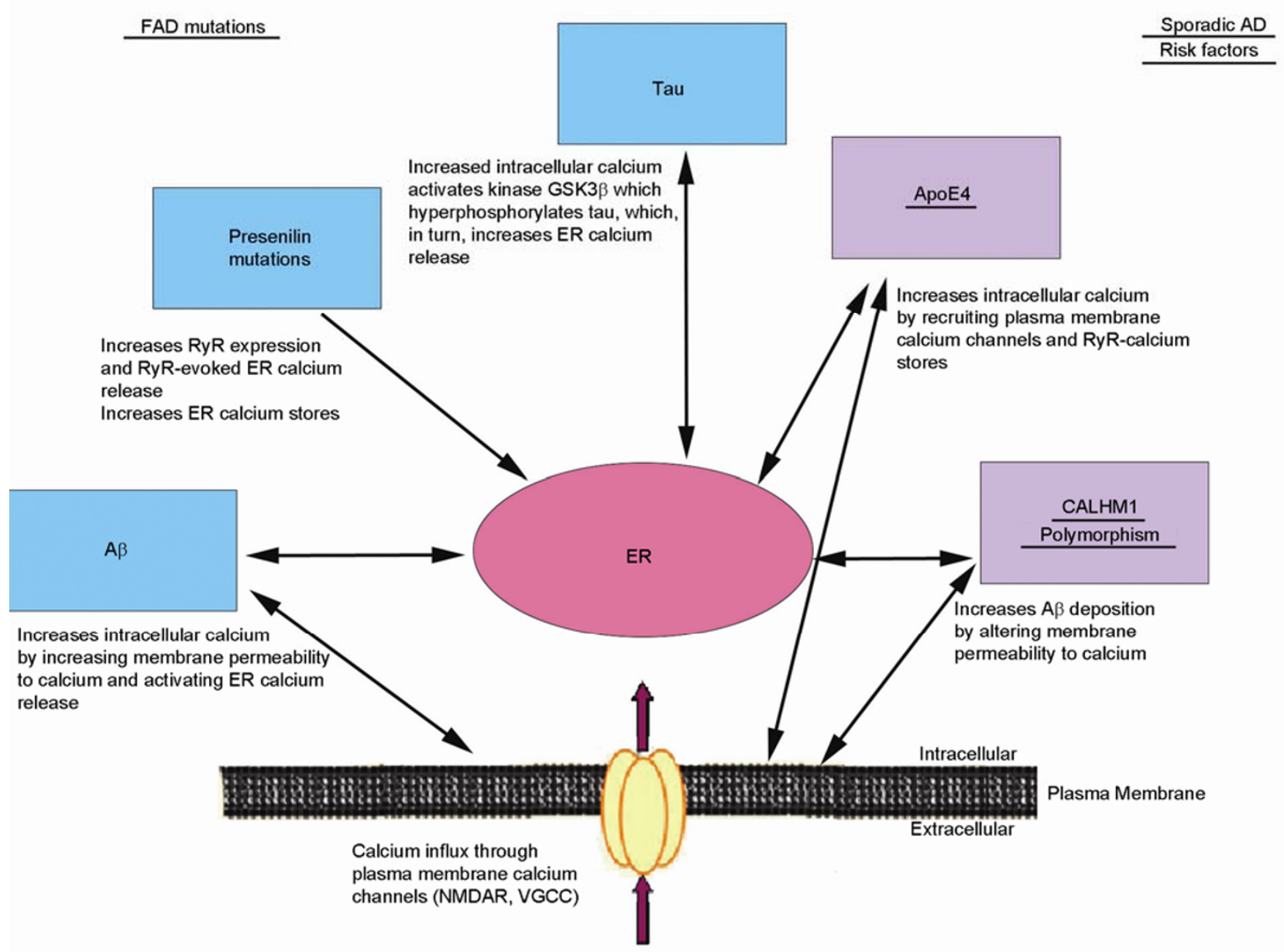

Figure 1 Convergent and enabling pathways for calcium involvement in the early pathogenesis of AD. Extracellular calcium influx through the plasma membrane or release from ER stores may be capable of inducing or facilitating several cardinal features of late-stage AD. These features in turn, can further increase ER calcium release, supporting a feedforward pathological cycle, resulting in gradually accumulating cellular insults. Ultimately, the cellular compensatory mechanisms may become overwhelmed and give way to pathological signaling cascades. (Reprinted with permission from Stutzmann, 2007).

\section{Calcium channels, neurotransmission and synaptic plasticity}

The dynamic interplay between calcium sources becomes particularly relevant when considering pre and postsynaptic mechanisms underlying neurotransmission and synaptic plasticity (Figure 2) [28,72-75]. Presynaptically, ER calcium mediates neurotransmitter release via coupling of calcium binding sensors to neurotransmitter vesicles. CICR through the RyR may trigger spontaneous neurotransmitter release that is detected as miniature postsynaptic potentials $[76,77]$. When presynaptic calcium levels are elevated in response to an action potential, the immediately releasable pool of neurotransmitter vesicles is released into the synaptic cleft. This is followed by a slow calcium-dependent phase during which the readily releasable pool is replenished from a reserve pool. CICR evoked by voltagedependent calcium entry can mobilize neurotransmitter vesicles from the reserve pool to the readily releasable pool and thus facilitate subsequent vesicle release [78,79]. This calcium-dependent release has implications for short-term forms of plasticity such as paired pulse facilitation (PPF), a form of presynaptic plasticity. PPF involves residual calcium remaining in the presynaptic terminal following the initial stimulus, thus increasing the probability of neurotransmitter release when a second stimulus in rapid temporal succession additively increases calcium influx into the presynaptic terminal. ER stores may be a source for the residual calcium that contributes to PPF, and conditions that enhance CICR, such as PS mutations, may alter vesicle release probabilities. Post-tetanic potentiation (PTP) is another form of presynaptic plasticity that occurs following a high frequency stimulus. PTP reflects an enhancement of neurotransmitter release that leads to strengthening of the synapse for a brief period of time, typically on the order of seconds to minutes. PTP is also thought to involve residual calcium that results from voltage-gated calcium influx that accompanies a high frequency stimulus, and CICR recruited by voltage-gated calcium influx can increase residual calcium 
$[75,77,79]$.

Postsynaptically, ER calcium initiates gene transcription [80], modulates membrane excitability and channel function $[81,82]$, and integrates with synaptically evoked responses to encode long term plasticity changes in neuron function $[83,84]$. Dendritic spines are typically the initial loci of synaptic plasticity and contain several calcium channels such as NMDAR, AMPAR, VGCC, RyR and $\mathrm{IP}_{3} \mathrm{R}$. Calcium in dendritic spines and dendrites regulates incoming electrochemical inputs for signal transmission through neurons, and depending on the combination of channels recruited, calcium influx can influence long-term changes in synaptic efficacy and plasticity. For example, NMDARmediated calcium entry into spines and dendrites is essential but not sufficient by itself for induction of LTP $[79,86]$. ER calcium stores can amplify the initial NMDAR-mediated signal and determine the polarity as well as input specificity of long-term plasticity. For example, blocking ER calcium stores eliminates LTD and results in a switch to LTP under the same stimulus conditions. Similarly, with an LTP-inducing stimulus, blocking ER stores reduces LTP while stimulating intracellular calcium release facilitates LTP $[56,87,88]$.

Certain forms of calcium-dependent synaptic plasticity, including LTP and LTD, are thought to underlie the cellular/molecular mechanisms of learning and memory [89-91]. This has been demonstrated in studies where NMDA calcium channels or calcium-dependent kinases required for synaptic plasticity were manipulated, and learning and memory deficits resulted. Specifically, NMDA receptors are required not just for induction of LTP but also for establishing the duration and maintenance of LTP via activation of protein kinase $\mathrm{M} \zeta(\mathrm{PKM} \zeta)$, an isoform of protein kinase C. When blocking NMDA receptors or PKM $\zeta$, LTP is abolished and spatial learning, particularly the generation and maintenance of new place fields, is disrupted [92-96]. Similarly, rodents lacking the essential NR1 subunit of the NMDA receptor demonstrate disrupted place cell function and are unable to locate a hidden platform in the Morris water maze, suggesting that calcium-permeable channels are crucial in spatial learning and memory [97].

Not only are changes in calcium levels important for transmission and plasticity induction, but the spatial and temporal patterns are critical encoding parameters as well. At synapses that support bidirectional plasticity, the induction of either LTP or LTD depends on the magnitude and temporal aspects of intracellular calcium dynamics-large and transient calcium rises are associated with LTP whereas modest and sustained calcium rises are associated with LTD $[86,88,98]$. Calcium/calmodulin-dependent protein kinase II (CAMKII) required for induction of LTP has low affinity for calcium and is activated when there are large but transient increases in postsynaptic calcium [99,100]. CAMKII together with protein kinase A (PKA) activate several downstream signaling cascades that drive AMPAR into the synapse [101-103] as well as CREB-dependent transcription of proteins that sustain synaptic plasticity [104]. On the other hand, calcineurin or protein phosphatase 2B ( $\mathrm{PP} 2 \mathrm{~B})$ associated with LTD has a much higher affinity for calcium and is activated by modest and sustained increases in postsynaptic calcium [105,106]. Activation of calcineurin leads to dephosphorylation and internalization of AMPA receptors from the synapse and therefore results in a reduction in synaptic strength [107-108].

The precise role of intracellular calcium stores in these processes is complex and may depend on the mechanism of calcium release from the ER (Figure 2). In neurons, the ER forms a continuous network that extends throughout the dendritic tree and into dendritic spines [109,110]. In CA1 pyramidal neurons, the ER in the soma and dendritic shafts express both $\mathrm{IP}_{3} \mathrm{R}$ and RyR, while ER networks in distal processes and dendritic spine heads express a greater proportion of RyR [80,112]. This suggests that calcium signaling involving these individual receptors may support different roles in synaptic activity-the $\mathrm{IP}_{3} \mathrm{Rs}$ may be involved in gene transcription and protein synthesis in the soma, while RyRs may be better positioned to modulate incoming synaptic activity directly in dendritic spine heads or subthreshold signals that are confined to the isolated geometry of the spine heads, whereas $\mathrm{IP}_{3} \mathrm{R}$ activation may enter extrasynaptic signaling pathways or require much higher threshold inputs. For example, in dendritic spines of hippocampal CA1 neurons, the NMDAR-mediated calcium signal is largely amplified by RyR-mediated CICR [78,113]. Blocking RyR with dantrolene obstructs the induction of LTP [56,114,115] as well as LTD (Chakroborty, unpublished findings), indicating that RyR-mediated ER calcium is required for sustained plasticity and by association, memory and related cognitive functions. Additional evidence for the fundamental role of RyR in synaptic plasticity emerges from studies using RyR knockout mice. For example, RyR3 knockout mice show enhanced LTP which is independent of NMDAR-mediated mechanisms, but also exhibit impaired LTD [116]. The RyR3 is expressed in dendritic processes of hippocampal neurons [60], suggesting that RyR3 isoform may function to suppress LTP and facilitate LTD. This may in turn serve to maintain the balance of excitation and inhibition that determines the overall stability of the synapse.

There also appears to be functional overlap between RyR and $\mathrm{IP}_{3} \mathrm{R}$-mediated calcium signaling and plasticity. Type 1 $\mathrm{IP}_{3} \mathrm{R}$ knockout mice also demonstrate enhanced LTP, while LTD is not affected [117], suggesting that $\mathrm{IP}_{3} \mathrm{R}$-sensitive ER calcium stores in general have an inhibitory role in LTP induction. Furthermore, $\mathrm{IP}_{3} \mathrm{R}$-mediated calcium stores outside dendritic spines may also suppress LTP in neighboring synapses, thus maintaining the input specificity that is characteristic of LTP. The differences in the effects of RyR and $\mathrm{IP}_{3} \mathrm{R}$-mediated calcium signaling on synaptic plasticity, especially LTD, may reflect their anatomical and kinetic 

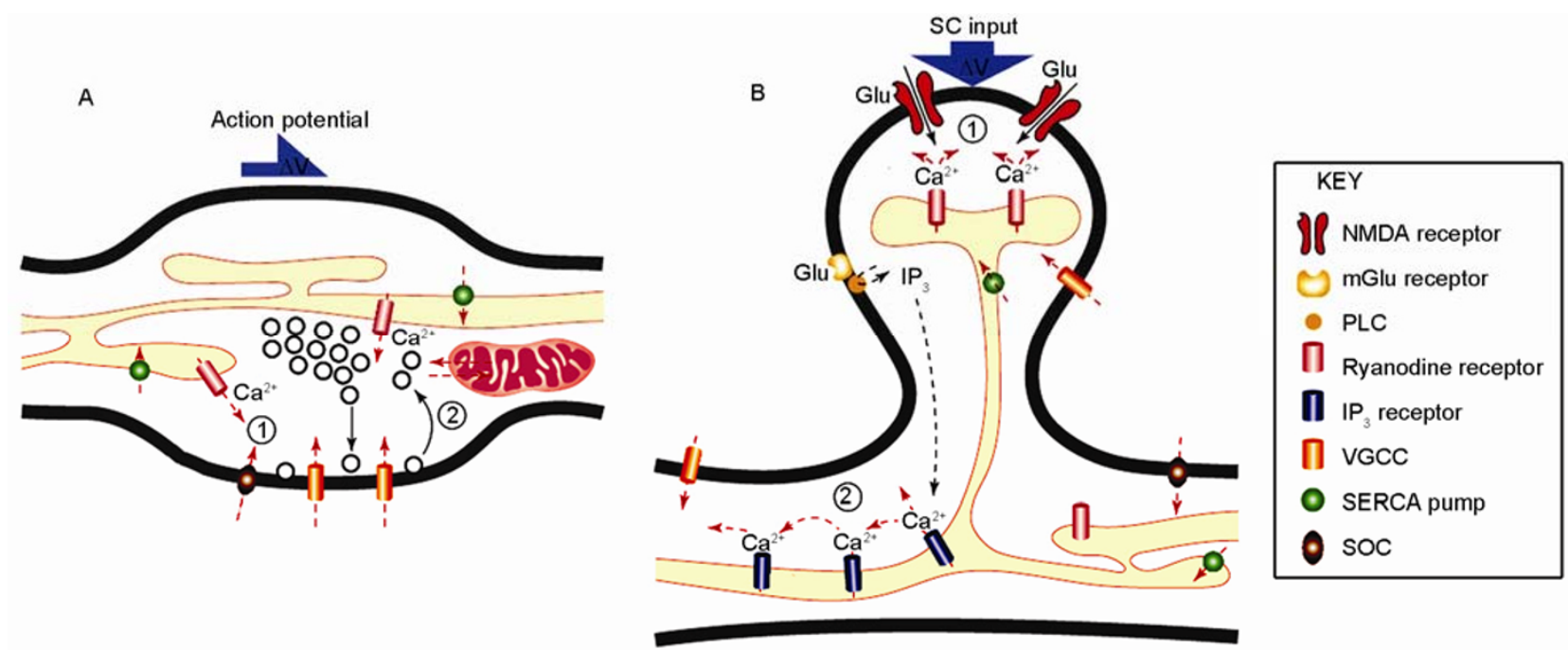

Figure 2 Possible ER calcium-dependent plasticity mechanisms in axon terminals and dendritic spines. A, at the presynaptic axon terminal-1) CICR can occur spontaneously to trigger synaptic vesicle release. During high frequency activity, CICR can be evoked by voltage-gated calcium influx to increase the residual calcium levels and release probability. (2) ER calcium release is implicated in endocytosis of fused vesicles to the reserve pool and mobilization of vesicles to the readily releasable pool. B, At the postsynaptic terminal-(1) NMDAR-mediated calcium signal is amplified by RyR in dendritic spines and is probably necessary for homosynaptic plasticity. (2) mGluR-mediated activation of the phospholipase C (PLC) pathway generates IP 3 . Subsequent activation of $\mathrm{IP}_{3} \mathrm{Rs}$ supports regenerative calcium waves, which may be involved in heterosynaptic plasticity and gene expression. (Reprinted with permission from Bardo et al., 2006).

differences in coupling to different calcium stores.

\section{Effects of altered ER calcium on neurophysi- ology: Implications for AD pathogenesis}

As implied in the preceding discussion, maintaining calcium homeostasis is crucial for synaptic activity and plasticity. Therefore, AD-linked mechanisms that interfere with calcium signaling are important to investigate from both a disease-manifesting and therapeutic standpoint. For example, PS mutations result in RyR-mediated ER calcium signaling disruptions which occur prior to $\mathrm{AD}$ histopathology and persist through old age $[32,46]$. Previous studies in $3 \times$ Tg-AD mice expressing mutant PS, APP and tau demonstrate synaptic dysfunction coincident with the detection of intracellular $A \beta$ deposits [44]. Yet prior to this stage, synaptic transmission appears normal. This is consistent with several studies in AD models suggesting that synaptic transmission is unaffected until amyloid deposits form $[25,118]$. Recent studies suggest that it is the soluble $\mathrm{A} \beta$ oligomers, and not insoluble amyloid plaques, that can cause synaptic dysfunction by disrupting intracellular calcium homeostasis via the formation of calcium-permeable channels [119-122]. The increased cytosolic calcium levels due to $A \beta$-calcium channels not only increase metabolic stress but also contribute to a decrease in spine density and dystrophy and aberrant sprouting of dendrites, resulting in synaptic dysfunction [123-125]. These studies have contributed to the view that $A \beta$ is the underlying mechanism driving synaptic dysfunction.

Yet, more detailed examination into the role of ER calcium stores at early stages, prior to $A \beta$ formation reveals pronounced deficits in baseline synaptic function that exist 'below the radar' of detection. A recent study in young $3 \times \mathrm{Tg}-\mathrm{AD}$ mice shows that dysregulated RyR-mediated ER calcium release alters hippocampal synaptic transmission and plasticity long before onset of AD histopathology (Figure 3) [56]. Under control conditions, basal synaptic transmission, PPF and LTP appear similar between the nontransgenic (NonTg) and AD mice. However, when the ER calcium contribution is manipulated, marked differences in the mechanisms underlying synaptic transmission and plasticity now emerge. Under conditions when the RyRs are blocked and the enhanced CICR effect is suppressed, the AD neurons now demonstrate enhanced basal synaptic transmission and altered short and long term plasticity, with no effects seen in the NonTg mice. For example, the I/O function and PPF were increased in AD neurons but remained unchanged in NonTg mice. This suggests that RyRmediated calcium has a prominent inhibitory effect in basal synaptic transmission and presynaptic neurotransmitter release in the AD mice but has little role in NonTg mice under these conditions. There are additional aberrant contributions of ER calcium to synaptic plasticity as well in the AD mice. In NonTg mice, RyR blockade had no effect on baseline responses, though LTP expression was reduced, a finding consistent with existing literature [126]. Yet, in AD mice, blocking RyR with dantrolene decreased baseline responses and produced a shift from LTP to modest LTD. Further di- 


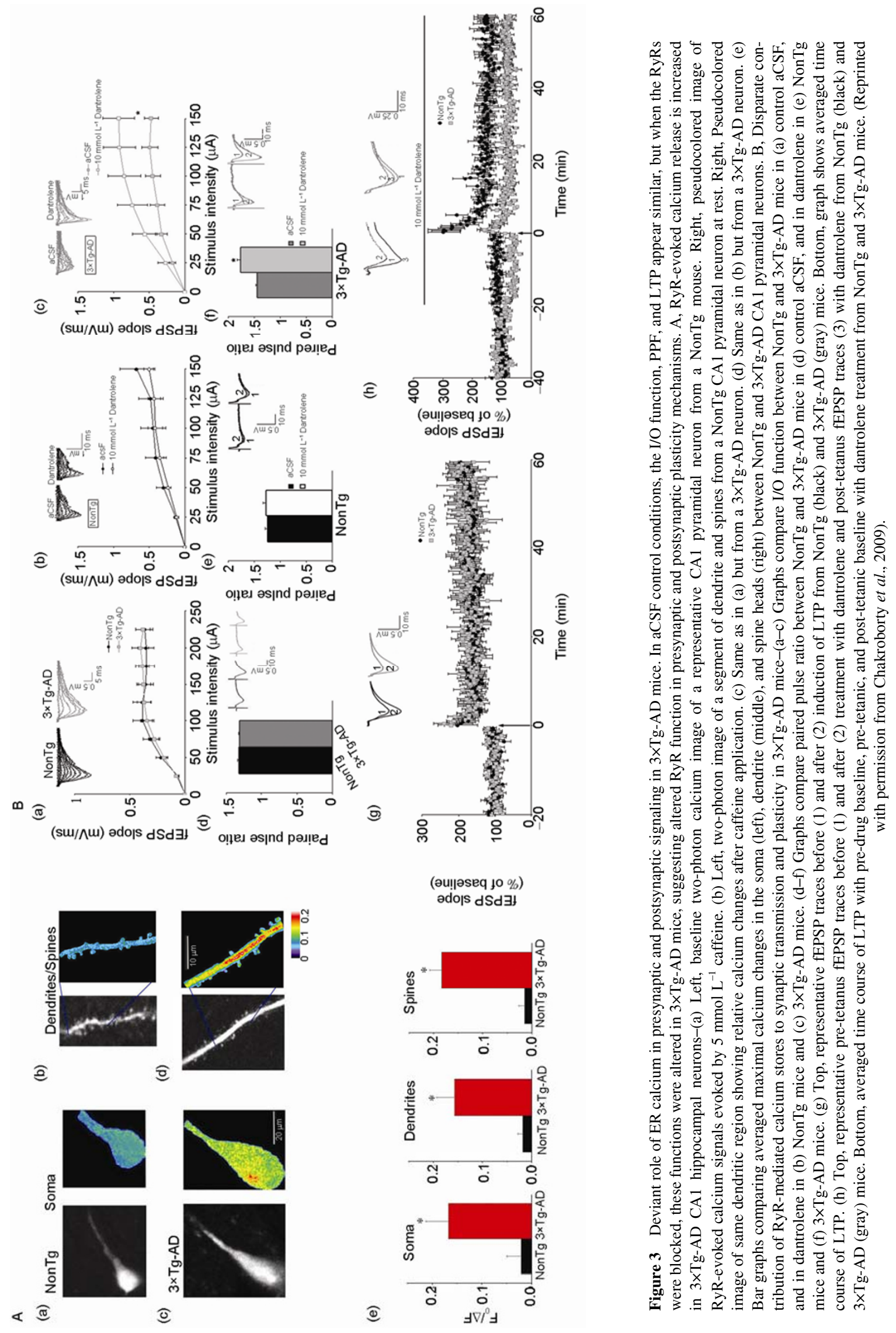


vergent effects of ER calcium contribution to plasticity are seen with LTD-generating paradigms. RyR blockade enhances LTD in AD mice, while completely abolishing LTD in NonTg mice (Chakroborty, unpublished data). Therefore, without RyR blockade, there appears to be no differences in synaptic transmission or plasticity between young AD and NonTg mice, suggesting that compensatory mechanisms may mask these alterations early on in the disease process but may break down as the disease progresses.

Consistent with these findings regarding the role of RyR-mediated calcium signaling in presynaptic transmission is another recent study utilizing conditional knockouts (cDKO) of PS1 and PS2 in either the pre or postsynaptic neurons at the Schaffer collateral synapse. Presenilins may modulate the role of presynaptic ER calcium in neurotransmitter release and in induction of LTP, and loss of PS function may result in dysfunctional presynaptic neurotransmission via a RyR-dependent mechanism. Conditional inactivation of PS in CA3 neurons from PS cDKO mice reduces neurotransmitter release, synaptic facilitation and markedly impairs LTP, while conditional inactivation of PS in postsynaptic CA1 neurons does not affect these functions [126]. RyR blockade also decreases synaptic facilitation in CA3 neurons of PS cDKO mice, suggesting that presynaptic deficits are mediated by RyR-sensitive calcium stores. These findings are further supported by earlier studies using PS1 knockout (KO) neurons as well as PS1 mutant mice. In these studies, PS1 mutant mice exhibit increased magnitude of LTP, while PS1 KO neurons are impaired in several calcium-dependent aspects of synaptic transmission [127,128]. These neurons have altered synaptic connections reflected by changes in spine morphology and spine density, and they show a redistribution of proteins critical for synaptic function such as PSD-95 and AMPA receptors. There is also upregulation of the PKA-cAMP signaling pathway that regulates CREB-dependent transcription, suggesting that these changes may ultimately impair synaptic transmission and plasticity, and therefore memory processes, in a manner that reflects upstream calcium signaling alterations.

\section{Summary}

Intracellular calcium signaling is crucial to neuronal function, synaptic transmission, and plasticity mechanisms underlying learning and memory. Owing to its ubiquitous role, sustained disruptions in calcium signaling have significant ramifications for neuronal and cognitive health over the lifetime of the organism. Current research suggests that the early disruptions in calcium homeostasis observed in AD incur pathogenic insults and are not inherent to normal aging $[32,46,129]$. Even though the direct mechanistic link between calcium dysregulation and $\mathrm{AD}$ pathology is still under investigation, one might surmise that calcium-mediated pathogenesis reflects a lifetime of gradually accumulating stressors and has multiple diffuse targets affecting many cellular systems. Eventually, these stressors become part of a feedforward pathological cascade that later facilitate $\mathrm{A} \beta$ and tau deposition, ER and mitochondrial stress, loss of synapses, and ultimately, loss of memory (Figure 1) [32,129-131]. Therefore, considering calcium dyshomeostasis as an integral component of AD-linked synaptic pathology may yield new insights into the cellular mechanisms of cognitive deficits and offer novel therapeutic interventions.

The authors would like to thank Ms. Kayla Ahlstrand and Ms. Corinne Schneider for editorial assistance in the preparation of this review.

1 Rossor M N, Fox N C, Freeborough P A, et al. Clinical features of sporadic and familial Alzheimer's disease. Neurodegeneration, 1996, 5: 393-397

2 LaFerla F M. Calcium dyshomeostasis and intracellular signaling in Alzheimer's disease. Nat Rev Neurosci, 2002, 3: 862-872

3 Selkoe D J. Alzheimer's disease: genes, proteins, and therapy. Physiol Rev, 2001, 81: 741-766

4 De Strooper B, Saftig P, Craessaerts K, et al. Deficiency of presenilin-1 inhibits the normal cleavage of amyloid precursor protein. Nature, 1998, 391: 387-390

5 Arriagada P V, Marzloff K, Hyman B T, Distribution of Alzheimer's-type pathologic changes in nondemented elderly individuals matches the pattern in Alzheimer's disease. Neurology, 1992, 42: 1681-1688

6 Kazee A M, Johnson E M. Alzheimer's disease pathology in non-demented elderly. J Alzheimer's Dis, 1998, 1: 81-89

7 Schmitt F A, Davis D G, Wekstein D R, et al. "Preclinical" AD revisited: neuropathology of cognitively normal older adults. Neurology, 2000, 55: 370-376

8 Goldman W P, Price J L, Storandt M et al. Absence of cognitive impairment or decline in preclinical Alzheimer's disease. Neurology, 2001, 56: 361-367

9 Lipton A M, Cullum C M, Satumtira S, et al. Contribution of asymmetric synapse loss to lateralizing clinical deficits in frontotemporal dementias. Arch Neurol, 2001, 58: 1233-1239

10 Selkoe DJ. Alzheimer's disease is a synaptic failure. Science, 2002, 298: 789-791

11 Coleman P D, Yao P J. Synaptic slaughter in Alzheimer's disease. Neurobiol Aging, 2003, 24: 1023-1027

12 Gylys K H, Fein J A, Yang F, et al. Synaptic changes in Alzheimer's disease: increased amyloid-beta and gliosis in surviving terminals is accompanied by decreased PSD-95 fluorescence. Am J Pathol, 2004, 165: 1809-1817

13 Scheff S W, Price D A. Synaptic pathology in Alzheimer's disease: a review of ultrastructural studies. Neurobiol Aging, 2003, 24: 1029-1046

14 Scheff S W, Price D A, Schmitt F A, et al. Hippocampal synaptic loss in early Alzheimer's disease and mild cognitive impairment. Neurobiol Aging, 2006, 27: 1372-1384

15 Pimplikar S W, Nixon R A, Robakis N K, et al. Mini-Symposium: Amyloid-independent mechanisms in Alzheimer's disease pathogenesis. J Neurosci, 2010, 30: 14946-14954

16 Goussakov I, Miller M B, Stutzmann G E. NMDA-mediated $\mathrm{Ca}^{2+}$ influx drives aberrant ryanodine receptor activation in dendrites of young Alzheimer's disease mice. J Neurosci, 2010, 30: 12128-12137

17 Hu N W, Ondrejcak T, Rowan M J. Glutamate receptors in preclinical research on Alzheimer's disease: Update on recent advances. Pharmacol Biochem Behav, 2011, In Press

18 Cerpa W, Dinamarca M C, Inestrosa N C. Structure-function implications in Alzheimer's disease: effect of Abeta oligomers at central 
synapses. Curr Alzheimer Res, 2008, 5: 233-243

19 Brion J P, Ando K, Heraud C, et al. Modulation of tau pathology in tau transgenic models. Biochem Soc Trans, 2010, 38: 996-1000

20 Gouras G, Tampellini D, Takahashi R, et al. Intraneuronal $\beta$-amyloid accumulation and synapse pathology in Alzheimer's disease. Acta Neuropathologica, 2010, 119: 523-541

21 Peethumnongsin E, Yang L, Kallhoff-Muñoz V, et al. Convergence of presenilin- and tau-mediated pathways on axonal trafficking and neuronal function. J Neurosci, 2010, 30: 13409-13418

22 Yuste R, Majewska A, Holthoff K. From form to function: calcium compartmentalization in dendritic spines. Nat Neurosci, 2000, 3: 653-659

23 Spruston N. Pyramidal neurons: dendritic structure and synaptic integration. Nat Rev Neurosci, 2008, 9: 206-221

24 Sorra K E, Harris K M. Overview on the structure, composition, function, development, and plasticity of hippocampal dendritic spines. Hippocampus, 2000, 10: 501-511

25 Zhang W, Benson D L. Development and molecular organization of dendritic spines and their synapses. Hippocampus, 2000, 10: 512-526

26 Zucker R S. Calcium- and activity-dependent synaptic plasticity. Curr Opin Neurobiol, 1999, 9: 305-313

27 Higley M J, Sabatini B L. Calcium signaling in dendrites and spines: practical and functional considerations. Neuron, 2008, 59: 902-913

28 Berridge M J. Neuronal calcium signaling. Neuron, 1998, 21: 13-26

29 Berridge M J, Lipp P, Bootman M D. The versatility and universality of calcium signaling. Mol Cell Biol, 2000, 1: 11-21

30 Mattson M P. Calcium and neurodegeneration. Aging Cell, 2007, 6: $337-350$

31 Bezprozvanny I. Calcium signaling and neurodegenerative diseases. Trends Mol Med, 2009, 15: 89-100

32 Stutzmann G E. The pathogenesis of Alzheimer's disease-is it a lifelong "calciumopathy"? Neuroscientist, 2007, 13: 546-559

33 Bezprozvanny I, Mattson M P. Neuronal calcium mishandling and the pathogenesis of Alzheimer's disease. Trends Neurosci, 2008, 15: 454-463

34 Foskett J K, White C, Cheung K H, et al. Inositol Trisphosphate Receptor $\mathrm{Ca}^{2+}$ Release Channels Physiol Rev, 2007, 87: 593-658

35 Finch E, Turner T, Goldin S. Calcium as a coagonist of inositol-1,4,5-trisphosphate-induced calcium release. Science, 1991, 252: 443-446

36 Friel D, Tsien R. A caffeine- and ryanodine-sensitive $\mathrm{Ca}^{2+}$ store in bullfrog sympathetic neurons modulates effects of $\mathrm{Ca}^{2+}$ entry on $\left[\mathrm{Ca}^{2+}\right]_{i}$. J Physiol, 1992, 450: 217-246

37 Verkhratsky A. The endoplasmic reticulum and neuronal calcium signaling. Cell Calcium, 2002, 32: 393-404

38 Gunter T, Yule D, Gunter, et al. Calcium and mitochondria. FEBS Letters, 2004, 567: 96-102

39 Tolar M, Keller J N, Chan S, et al. Truncated apolipoprotein E (ApoE) causes increased intracellular calcium and may mediate ApoE neurotoxicity. J Neurosci, 1999, 19: 7100-7110

40 Dreses-Werringloer U, Lambert J C, Vingtdeux V, et al. A polymorphism in CALHM1 influences $\mathrm{Ca}^{2+}$ homeostasis, $\mathrm{A} \beta$ levels, and Alzheimer's disease risk. Cell, 2008, 133: 1149-1161

41 Ito $\mathrm{E}$, Oka $\mathrm{K}$, Etcheberrigaray $\mathrm{R}$, et al. Internal $\mathrm{Ca}^{2+}$ mobilization is altered in fibroblasts from patients with Alzheimer's disease. Proc Natl Acad Sci, 1994, 91: 534-538

42 Guo Q, Furukawa K, Sopher B L, et al. Alzheimer's PS-1 mutation perturbs calcium homeostasis and sensitizes PC12 cells to death induced by amyloid beta peptide. Neuroreport, 1996, 8: 379-383

43 Leissring M A, Paul B A, Parker I, et al. Alzheimer's presenilin-1 mutation potentiates inositol 1,4,5-triphosphate-mediated calcium signaling in Xenopus oocytes. J Neurochem, 1999, 72: 1061-1068

44 Oddo S, Caccamo A, Shepherd J D, et al. Triple-transgenic model of Alzheimer's disease with plaques and tangles: intracellular $A \beta$ and synaptic dysfunction. Neuron, 2003, 39: 409-421

45 Stuzmann G E, Caccamo A, LaFerla F M, et al. Dysregulated $\mathrm{IP}_{3}$ signaling in cortical neurons of knock-in mice expressing an $\mathrm{Alz}$ heimer's-linked mutation in presenilin 1 results in exaggerated $\mathrm{Ca}^{2+}$ signals and altered membrane excitability. J Neurosci, 2004, 24:
508-513

46 Stutzmann G E, Smith I, Caccamo A, et al. Enhanced ryanodine receptor recruitment contributes to $\mathrm{Ca}^{2+}$ disruptions in young, adult, and aged Alzheimer's disease mice. J Neurosci, 2006, 26: 5180-5189

$47 \mathrm{Tu} \mathrm{H}$, Nelson O, Bezprozvanny A, et al. Presenilins form ER $\mathrm{Ca}^{2+}$ leak channels, a function disrupted by familial Alzheimer's disease-linked mutations. Cell, 2006, 126: 981-993

48 Nelson O, Tu H, Lei T, et al. Familial Alzheimer disease-linked mutations specifically disrupt $\mathrm{Ca}^{2+}$ leak function of presenilin 1 . J Clin Invest, 2007, 117: 1230-1239

49 Cheung K H, Shineman D, Müller M, et al. Mechanism of $\mathrm{Ca}^{2+}$ disruption in Alzheimer's disease by presenilin regulation of $\mathrm{InsP}_{3}$ receptor channel gating. Neuron, 2008, 58: 871-883

50 Cheung K, Mei L, Mak D, et al. Gain-of-function enhancement of IP3 receptor modal gating by familial Alzheimer's disease-linked presenilin mutants in human cells and mouse neurons. Sci Signal, 2010, 3: 22-30

51 Smith I F, Hitt B, Green K N, et al. Enhanced caffeine-induced $\mathrm{Ca}^{2+}$ release in the $3 \times \mathrm{Tg}-\mathrm{AD}$ mouse model of Alzheimer's disease. J Neurochem, 2005, 94: 1711-1718

52 Koizumi S, Lipp P, Berridge M J, et al. Regulation of ryanodine receptor opening by lumenal $\mathrm{Ca}^{2+}$ underlies quantal $\mathrm{Ca}^{2+}$ release in PC12 cells. J Biol Chem, 1999, 274: 33327-33333

53 Berridge M J. The endoplasmic reticulum: a multifunctional signaling organelle. Cell Calcium, 2002, 32: 235-249

54 Rybalchenko V, Hwang S Y, Rybalchenko N, et al. The cytosolic $\mathrm{N}$-terminus of presenilin-1 potentiates mouse ryanodine receptor single channel activity. Int J Biochem Cell Biol, 2008, 40: 84-97

55 Chan S L, Mayne M, Holden C P, et al. Presenilin-1 mutations increase levels of ryanodine receptors and calcium release in $\mathrm{PC} 12$ cells and cortical neurons. J Biol Chem, 2000, 275: 18195-18200

56 Chakroborty S, Goussakov I, Miller M B, et al. Deviant ryanodine receptor-mediated calcium release resets synaptic homeostasis in presymptomatic 3×Tg-AD mice. J Neurosci, 2009, 29: 9458-9470

57 Supnet C, Grant J, Kong H, et al. Amyloid-beta- ${ }_{(1-42)}$ increases ryanodine receptor-3 expression and function in neurons of TgCRND8 mice. J Biol Chem, 2006, 281: 38440-38447

58 Supnet C, Noonan C, Richard J, et al. Up-regulation of the type 3 ryanodine receptor is neuroprotective in the TgCRND8 mouse model of Alzheimer's disease. J Neurochem, 2010, 112: 356-365

59 Lai F A, Dent M, Wickenden C, et al. Expression of a cardiac $\mathrm{Ca}^{2+}$-release channel isoform in mammalian brain. Biochem J, 1992, 288: 553-564

60 Hertle D N, Yeckel M F. Distribution of inositol-1,4,5-trisphosphate receptor isotypes and ryanodine receptor isotypes during maturation of the rat hippocampus. Neuroscience, 2007, 150: 625-638

61 Bruno A, Huang J, Bennett D A, et al. Altered ryanodine receptor expression in mild cognitive impairment and Alzheimer's disease. Neurobiology of Aging, 2011, In Press

62 Demuro A, Mina E, Kayed R, et al. Calcium dysregulation and membrane disruption as a ubiquitous neurotoxic mechanism of soluble amyloid oligomers. J Biol Chem, 2005, 280: 17294-17300

63 Glabe C C. Amyloid accumulation and pathogenesis of Alzheimer's disease: significance of monomeric, oligomeric and fibrillar Abeta. Subcell Biochem, 2005, 38: 167-177

64 Deshpande A, Mina E, Glabe C G, et al. Different conformations of amyloid beta induce neurotoxicity by distinct mechanisms in human cortical neurons. J Neurosci, 2006, 26: 6011-6018

65 Pierrot N, Ghisdal P, Caumont A S, et al. Intraneuronal amyloid-beta-42 production triggered by sustained increase of cytosolic calcium concentration induces neuronal death. J Neurochem, 2004, 88: $1140-1150$

66 McGowan E, Pickford F, Kim J, et al. Abeta 42 is essential for parenchymal and vascular amyloid deposition in mice. Neuron, 2005, 47: 191-199

67 Querfurth H W, Selkoe D J. Calcium ionophore increases amyloid beta peptide production by cultured cells. Biochemistry, 1994, 33: 4550-4561

68 Avila J, Perez M, Lim F, et al. Tau in neurodegenerative diseases: tau 
phosphorylation and assembly. Neurotox Res, 2004, 6: 477-482

69 Corder E H, Saunders A M, Strittmatter W J, et al. Gene dose of apolipoprotein E type 4 allele and risk of Alzheimer's disease in late onset families. Science, 1993, 261: 921-923

70 Farrer L A, Cupples L A, Haimes J L, et al. Effects of age, sex and ethnicity on the association between apolipoprotein $\mathrm{E}$ genotype and Alzheimer's disease. A meta-analysis. APOE and Alzheimer's disease Meta Analysis Consortium. JAMA, 1997, 278: 1349-1356

71 Namba Y, Tomonaga M, Kawasaki H et al. Apolipoprotein E immunoreactivity in cerebral amyloid deposits and neurofibrillary tangles in Alzheimer's disease and kuru plaque amyloid in Creutzfeldt-Jacob disease. Brain Res, 1991, 541: 163-166

72 Toescue E C, Verkhratsky A. Neuronal aging from an intraneuronal perspective: roles of endoplasmic reticulum and mitochondria. Cell Calcium, 2003, 34: 311-323

73 Park M K, Choi Y M, Kang Y K, et al. The endoplasmic reticulum as an integrator of multiple dendritic events. Neuroscientist, 2008, 14: 68-77

74 Emptage N J, Reid C A, Fine A. Calcium stores in hippocampal synaptic boutons mediate short-term plasticity, store-operated $\mathrm{Ca}^{2+}$ entry, and spontaneous transmitter release. Neuron, 2001, 29: 197-208

75 Zucker R S, Regehr W G. Short-term synaptic plasticity. Annu Rev Physiol, 2002, 64: 355-405

76 Kuromi H, Kidokoro Y. Selective replenishment of two vesicle pools depends on the source of $\mathrm{Ca}^{2+}$ at the Drosophila synapse. Neuron, 2002, 35: 333-343

77 Bouchard R, Pattarini R, Geiger J D. Presence and functional significance of presynaptic ryanodine receptors. Prog Neurobiol, 2003, 69: $391-418$

78 Emptage N J, Bliss T V, Fine A. Single synaptic events evoke NMDA receptor-mediated release of calcium from internal stores in hippocampal dendritic spines. Neuron, 1999, 22: 115-124

79 Bardo S, Cavazzini M G, Emptage N J. The role of endoplasmic reticulum calcium stores in the plasticity of central neurons. Trends Pharmacol Sci, 2006, 27: 78-84

80 Mellström B, Naranjo J R. Mechanisms of $\mathrm{Ca}^{2+}$-dependent transcription. Curr Opin Neurobiol, 2001, 11: 312-319

81 Watanabe S, Hong M, Lasser-Ross N, et al. Modulation of calcium wave propagation in the dendrites and to the soma of rat hippocampal pyramidal neurons. J Physiol, 2006, 575: 455-468

82 Stutzmann G E, LaFerla F M, Parker I. $\mathrm{Ca}^{2+}$ signaling in mouse cortical neurons studied by two-photon imaging and photoreleased inositol triphosphate. J Neurosci, 2003, 23: 758-765

83 Hagenston A M, Fitzpatrick J S, Yeckel M F. mGluR-mediated calcium waves that invade the soma regulate firing in layer $\mathrm{V}$ medial prefrontal cortical pyramidal neurons. Cereb Cortex, 2008, 18: 407-423

84 Ross W N, Nakamura T, Watanabe S, et al. Synaptically activated $\mathrm{Ca}^{2+}$ release from internal stores in CNS neurons. Cell Mol Neurobiol, 2005, 25: 283-295

85 Yasuda R, Sabatini B L, Svoboda K. Plasticity of calcium channels in dendritic spines. Nat Neurosci, 2003, 6: 948-955

86 Fitzjohn S M, Collingridge G L. Calcium stores and synaptic plasticity. Cell Calcium, 2002, 32: 405-411

87 Raymond C R, Redman S J. Spatial segregation of neuronal calcium signals encodes different forms of LTP in rat hippocampus. J Physiol, 2006, 570: 97-111

88 Nishiyama M, Hong K, Mikoshiba K, et al. Calcium stores regulate the polarity and input specificity of synaptic modification. Nature, 2000, 408: 584-588

89 Bliss T V, Collingridge G L. A synaptic model of memory: LTP in the hippocampus. Nature, 1993, 361: 31-39

90 Martin S J, Grimwood P D, Morris R G M. Synaptic plasticity and memory: An evaluation of the hypothesis. Annu Rev Neurosci, 2000, 23: 649-711

91 Whitlock J R, Heynen A J, Shuler M G, et al. Learning induces long-term potentiation in the hippocampus. Science, 2006, 313: 1093-1097

92 Villarreal D M, Do V, Haddad E, et al. NMDA receptor antagonists sustain LTP and spatial memory: active processes mediate LTP decay. Nat Neurosci, 2001, 5: 48-52

93 Malenka R C, Bear M F. LTP and LTD: an embarrassment of riches. Neuron, 2004, 44: 5-21

94 Ling D S, Benardo L S, Serrano P A, et al. Protein kinase Mzeta is necessary and sufficient for LTP maintenance. Nat Neurosci, 2002, 5: 295-296

95 Morris R G M, Frey U. Hippocampal synaptic plasticity: role in spatial learning or the automatic recording of attended experience? Philos Trans R Soc Lond B Biol Sci, 1997, 352: 1489-1503

96 Pastalkova E, Serrano P, Pinkhasova D, et al. Storage of spatial information by the maintenance mechanism of LTP. Science, 2006, 313: 1141-1144

97 Tsien J Z, Chen D F, Gerber D, et al. Subregion- and cell type-restricted gene knockout in mouse brain. Cell, 1996, 87: 1317-1326

98 Cummings J A, Mulkey R M, Nicoll R A, et al. $\mathrm{Ca}^{2+}$ signaling requirements for long-term depression in the hippocampus. Neuron. 1996, 16: 825-833

99 Foster T C. Calcium homeostasis and modulation of synaptic plasticity in the aged brain. Aging Cell, 2007; 6: 319-325

100 Lisman J, Schulman H, Cline H. The molecular basis of CAMKII function in synaptic and behavioral memory. Nat Rev Neurosci, 2002, 3: 175-190

101 Hayashi Y, Shi S H, Esteban J A, et al. Driving AMPA receptors into synapses by LTP and CAMKII: requirement for GluR1 and PDZ domain interaction. Science, 2000, 287: 2262-2267

102 Malinow R, Malenka R C. AMPA receptor trafficking and synaptic plasticity. Annu Rev Neurosci, 2002, 25: 103-126

103 Bredt D S, Nicoll R A. AMPA receptor trafficking at excitatory synapses. Neuron, 2003, 40: 361-379

104 Bito H, Takemoto-Kimura S. $\mathrm{Ca}^{2+} / \mathrm{CREB} / \mathrm{CBP}-$ dependent gene regulation: a shared mechanism critical in long-term synaptic plasticity and neuronal survival. Cell Calcium, 2003, 34: 25-30

105 Mulkey R M, Malenka R C. Mechanisms underlying induction of homosynaptic long-term depression in area CA1 of the hippocampus. Neuron, 1992: 9: 967-975

106 Mulkey R M, Endo S, Shenolikar S, et al. Involvement of a calcineurin/inhibitor-1 phosphatase cascade in hippocampal long-term depression. Nature, 1994, 369: 486-488

107 Beattie E C, Carroll R C, Yu X, et al. Regulation of AMPA receptor endocytosis by a signaling mechanism shared with LTD. Nat Neurosci, 2000, 3: 1291-1300

108 Lee S H, Liu L, Wang Y T et al. Clathrin adaptor AP2 and NSF interact with overlapping sites of GluR2 and play distinct roles in AMPA receptor trafficking and hippocampal LTD. Neuron, 2002, 36: 661-674

109 Harris K M, Stevens J K, Dendritic spines of CA1 pyramidal cells in the rat hippocampus: serial electron microscopy with reference to their biophysical characteristics. J Neurosci, 1989, 9: 2982-2997

110 Spacek J, Harris K M. Three dimensional organization of smooth endoplasmic reticulum in hippocampal CA1 dendrites and dendritic spines of immature and mature rat. J Neurosci, 1997, 17: 190-203

111 Sharp A H, McPherson P S, Dawson T M, et al. Differential immunohistochemical localization of inositol-1,4,5-trisphosphate- ad ryanodine-sensitive $\mathrm{Ca}^{2+}$ release in channels in rat brain. $\mathrm{J}$ Neurosci, 1993, 13: 3051-3063

112 Nakamura T, Barbara J G, Nakamura K, et al. Synergistic release of $\mathrm{Ca} 2+$ from IP3-sensitive stores evoked by synaptic activation of mGluRs paired with backpropagating action potentials. Neuron, 1999, 24: 727-737

113 Alford S, Frenguelli B G, Schofield J G, et al. Characterization of $\mathrm{Ca}^{2+}$ signals induced in hippocampal CA1 neurons by the synaptic activation of NMDA receptors. J Physiol, 1993, 469: 693-716

114 Obenaus A, Mody I, Baimbridge K G. Dantrolene-Na (Dantrium) blocks induction of long-term potentiation in hippocampal slices. Neurosci Lett, 1989, 98: 172-178

115 Harvey J, Collingridge G L. Thapsigargin blocks the induction of long-term potentiation in rat hippocampal slices. Neurosci Lett, 1992, 
139: 197-200

116 Futatsugi A, Kato K, Ogura $\mathrm{H}$, et al. Facilitation of NMDARindependent LTP and spatial learning in mutant mice lacking ryanodine receptor type 3. Neuron, 1999, 24: 701-713

117 Fujii S, Matsumoto M, Igarashi K, et al. Synaptic plasticity in hippocampal CA1 neurons of mice lacking type 1 inositol-1,4,5-triphosphate receptors. Learn Mem, 2000, 7: 312-320

118 Rowan M J, Klyubin I, Cullen W K, et al. Synaptic plasticity in animal models of early Alzheimer's disease. Philos Trans R Soc Lond B Biol Sci, 2003, 358: 821-828

119 Kawahara M, Kuroda Y. Molecular mechanism of neurodegeneration induced by Alzheimer's beta-amyloid protein: channel formation and disruption of calcium homeostasis. Brain Res Bull, 2000, 53: 389-397

120 Selkoe D J. Soluble oligomers of the amyloid beta-protein impair synaptic plasticity and behavior. Behav Brain Res, 2008, 192: 106-113

121 Shankar G M, Li S, Mehta T H, et al. Amyloid-beta protein dimers isolated directly from Alzheimer's brains impair synaptic plasticity and memory. Nat Med, 2008, 14: 837-842

122 Lin H, Bhatia R, Lal R. Amyloid beta protein forms ion channels: implications for Alzheimer's disease pathophysiology. FASEB J, 2001, 15: 2433-2444

123 Mattson M P, Chan S L. Neuronal and glial calcium signaling in Alzheimer's disease. Cell Calcium, 2003, 34: 385-397
124 Kuchibhotla K V, Goldman S T, Lattarulo C R, et al. A $\beta$ plaques lead to aberrant regulation of calcium homeostasis in vivo resulting in structural and functional disruption of neuronal networks. Neuron, 2008, 59: 214-225

125 Koffie RM, Meyer-Luehmann M, Hashimoto T, et al. Oligomeric amyloid $\beta$ associates with postsynaptic densities and correlates with excitatory synapse loss near senile plaques. Proc Natl Acad Sci, 2009, 106: 4012-4017

126 Zhang C, Wu B, Beglopoulos V, et al. Presenilins are essential for regulating neurotransmitter release. Nature, 2009, 460: 632-636

127 Parent A, Linden D J, Sisodia S S, et al. Synaptic transmission and hippocampal long-term potentiation in transgenic mice expressing FAD-linked presenilin 1. Neurobiol Dis, 1999, 6: 56-62

128 Parent A T, Barnes N Y, Taniguchi Y, et al. Presenilin attenuates receptor-mediated signaling and synaptic function. J Neurosci, 2005, 25: $1540-1549$

129 Verkhratsky A. Physiology and pathophysiology of calcium store in the endoplasmic reticulum of neurons. Physiol Rev, 2005, 85: 201-279

130 Mattson M P, Gary D S, Chan S L, et al. Perturbed endoplasmic reticulum function, synaptic apoptosis and the pathogenesis of Alzheimer's disease. Biochem Soc Symp, 2001, 67: 151-162

131 Mattson M P. ER calcium and Alzheimer's disease: in a state of flux. Sci Signal, 2010, 3: pe10

Open Access This article is distributed under the terms of the Creative Commons Attribution License which permits any use, distribution, and reproduction in any medium, provided the original author(s) and source are credited. 